Abstract ID: 53

\title{
Antibiotic susceptibility study of metal-gentamicin complexes against Pseudomonas aeruginosa biofilms
}

\author{
Intan Azura Shahdan ${ }^{\mathrm{a}}$ | Fatimah Zahrah Mohd Sobri ${ }^{\mathrm{a}}$ | Mohammad Faiz Hizzuan Hanapi ${ }^{\mathrm{b}}$ | Hanani Ahmad \\ Yusof $^{\text {a }}$ | Fiona N.-F. How \\ ${ }^{a}$ Department of Biomedical Sciences, Kulliyyah of Allied Health Sciences, International Islamic \\ University Malaysia \\ ${ }^{b}$ Department of Chemistry, Kulliyyah of Science, International Islamic University Malaysia
}

Introduction: Inherent resistance of biofilm bacteria to conventional antibiotics is alarming because biofilms induce antibiotic resistance to an order of three or more in magnitude greater than those displayed by planktonic bacteria. Pseudomonas aeruginosa is an infectious organism that causes the hallmark of chronic infections including hospital acquired infections that leads to high morbidity and mortality. One of the reasons for the occurrence of resistance is its ability to form biofilms. In this study, the resistance of $P$. aeruginosa biofilms against a series of metal-antibiotics, an alternative to the conventional antibiotics, was investigated. Methods: A series of metal-antibiotic complexes derived from gentamicin was synthesized to give metal-gentamicin complexes. The metal contents of all the compounds were determined using Atomic Absorption Spectroscopy (AAS). Antibiotic susceptibility testing of the gentamicin-antibiotic complexes against the biofilms was conducted using broth microdilution assay. Results: The results showed that $P$. aeruginosa is susceptible against all the metal-gentamicin complexes, which include, Ni(II), Fe(II), $\mathrm{Cu}(\mathrm{II}), \mathrm{Zn}(\mathrm{II})$ and $\mathrm{Co}(\mathrm{II})$ complexes; all were tested at 0.25 to $1 \mathrm{mmol}$ concentrations. Conclusions: The results show that all metal-gentamicin complexes have higher antimicrobial activity than gentamicin $(0.25$ to $1 \mathrm{mmol}$ ) on its own. Finally, mechanisms of $P$. aeruginosa biofilms resistance to these metal-antibiotics are also proposed.

KEYWORDS: antibiotic resistance, biofilms, Pseudomonas aeruginosa, metal-antibiotics complexes 